\section{Ciencia Económica}

Revista electrónica de la Facultad de Economía de la Universidad Nacional Autónoma de México

UNAM

Enrique Graue Wiechers

Rector

Leonardo Lomelí Vanegas

Secretario General

Leopoldo Silva Gutiérrez

Secretario Administrativo

Alberto Ken Oyama Nakagawa

Secretario de Desarrollo Institucional

César Iván Astudillo Reyes

Secretario de Atención

a la Comunidad Universitaria

Mónica González Contró

Abogada General

FACULTAD DE ECONOMÍA

Eduardo Vega López Director

Rosa Carmina Ramírez Contreras Secretaria General

Porfirio Díaz Rodríguez

Secretario Administrativo

Ricardo Iglesias Flores

Coordinador de Publicaciones

CIENCIA ECONÓMICA

Mauro Rodríguez García Director

Karina Navarrete Pérez

Secretaria Técnica

Comité Editorial

Andrés Blancas Neria (Instituto de Investigaciones Económicas, UNAM)

Jorge Ibarra Consejo

(Facultad de Economía, UNAM)

Carlos Ibarra Niño

(Universidad de las Américas, Puebla)

Carlos Maya Ambía

(Universidad de Guadalajara)

Gustavo Vargas Sánchez

(Facultad de Economía, UNAM)

Fundadores

Jorge Carreto Sanguinés Irma Escárcega Aguirre Rogelio Huerta Quintanilla Mauro Rodríguez García Paulo Scheinvar Akcelrad† doi: 10.22201/fe.24484962e.2015.v4n6.a4
Facultad de Economía

\section{Año $4 \cdot$ no. 6 enero-junio de 2015}

Fecha de publicación: 2 de febrero de 2016

\title{
Reflexiones sobre la docencia
}

\begin{abstract}
Escárcega Aguirre, I., 2015. Comprensión lectora y rendimiento escolar en estudiantes de la Facultad de Economía de la UNAM. Ciencia
\end{abstract} Económica, 4(6), enero-junio, pp. 79-102.
Ciencia Económica • Publicación semestral Revista electrónica de la Facultad de Economía de la UNAM Academia de Teoría Económica 


\section{Comprensión lectora y rendimiento escolar en estudiantes de la Facultad de Economía de la UNAM}

Irma Escárcega Aguirre

Facultad de Economía, Universidad Nacional Autónoma de México (UNAM) <iescarce@hotmail.com>

doi: 10.22201/fe.24484962e.2015.v4n6.a4

\section{INTRODUCCIÓN}

D iferentes autores han señalado la importancia de la lectura como el primer acercamiento al aprendizaje. Malmquist ${ }^{1}$ señala: "los hábitos intelectuales y perceptivos motrices que se adquieren en el aprendizaje de la lectura nos marca para toda la vida". Otros consideran un obstáculo insalvable las deficiencias del niño en la lectura, de manera que si en el aprendizaje de la lectura no se contempla la comprensión de lo que se lee, ese niño no será capaz de entender más adelante otras lecturas. ${ }^{2}$ Algunos más consideran que a pesar de las deficiencias en la lectura en los niveles básicos, es posible superar el problema, siempre y cuando se atienda, pero "la lectura que mayormente se promueve en las escuelas mutila el desarrollo humano antes que potenciarlo". ${ }^{3}$

Entre los investigadores existe acuerdo en que la complejidad del proceso de comprensión lectora tiene una connotación individual y, por tanto, da lugar a diferencias importantes en los niveles que se alcanzan. También hay acuerdo en que la enseñanza de la lectura con frecuencia se enfoca a lograr la fluidez y rapidez necesaria para una adecuada decodificación, pero es menos frecuente que se insista en el procesamiento de los textos hasta alcanzar la comprensión lectora.

La mayoría de los autores que han analizado el caso mexicano concluyen que la estructura del sistema educativo en el nivel primario incorpora como preocupación central la lectura en los primeros tres grados, pero no se alcanza el nivel de comprensión lectora.

1 Citado por Ramos Maldonado (2005, p. 23).

2 Ibid., p. 23.

3 Ibid., p. 100. 
Las causas de esta situación son diversas. Para algunos autores, el contenido de los programas de estudio en la materia de español es demasiado extenso, compite, además, con los contenidos del resto de las materias. Para otros, el problema radica en que la enseñanza de la lectura en el ámbito escolar no abarca todas las etapas que corresponden al proceso lector, queda inconcluso y, por tanto, repercutirá en las capacidades de aprendizaje en periodos posteriores de la vida académica, pero también en las habilidades laborales. Otros más señalan como principal problema las limitaciones de los profesores en este campo.

Lo cierto es que a nivel universitario son frecuentes las quejas de los docentes respecto a la capacidad de los estudiantes para entender y procesar las lecturas de los textos que corresponden a sus asignaturas, lo cual se expresa en una frase como "los estudiantes no saben leer". ${ }^{4} \mathrm{~A}$ este problema se le atribuye una buena parte de las dificultades en la acreditación de una asignatura. La otra, a las deficiencias en el manejo de las matemáticas que, para nuestro caso de estudio, resulta en elevados índices de reprobación.

Surgen, entonces, algunas preguntas: ¿ existe alguna relación entre la comprensión lectora y la capacidad de aprendizaje?, ¿es posible medir el grado de relación?, ¿hay algunos factores determinantes en la comprensión lectora? Estas preguntas intentarán resolverse a lo largo de un estudio sobre la relación entre la comprensión lectora y el rendimiento escolar en los estudiantes de la Facultad de Economía de la Universidad Nacional Autónoma de México (UNAM).

Para abordar el problema se analizaron diferentes enfoques teóricos sobre el proceso de la lectura, donde destacan la teoría transaccional y la psicología sociocultural como los enfoques que permiten un mayor avance en la complejidad del proceso.

En cuanto a la obtención de datos, se consideró adecuada la realización de una investigación para una muestra de una generación reciente de estudiantes de la Facultad de Economía. Con ello se pretendió establecer el nivel de correlación causal, que en principio señala la comprensión lectora como variable independiente y el nivel de aprovechamiento como variable dependiente.

La primera variable se obtuvo de los resultados de las evaluaciones que la Dirección General de Evaluación Educativa (DGEE) de la UNAM pro-

4 Esta expresión tiene como fuente un sondeo que realizó la autora a una muestra de alrededor de 30 profesores de la Facultad de Economía respecto a los problemas que, de acuerdo a su opinión, presentan los estudiantes en sus grupos. 
porcionó. Para los niveles de aprovechamiento se tomaron dos elementos: las calificaciones de los estudiantes de la muestra y el número de materias acreditadas y no acreditadas por semestre. La investigación se complementa con un cuestionario, de elaboración propia con orientación de la literatura sobre el proceso lector, aplicado a los estudiantes de la muestra, cuyo propósito es buscar elementos de análisis sobre las prácticas de lectura y las estrategias de estudio.

En este trabajo presentamos las primeras conclusiones derivadas del análisis de los datos obtenidos tanto de la evaluación de la DGEE como de los cuestionarios y de las calificaciones.

Partiendo de la información general y de la revisión teórica sobre la importancia y problemas de la lectura, se plantearon las siguientes hipótesis generales y particulares.

\section{Hipótesis general}

El nivel de aprovechamiento escolar es el resultado de diferentes factores: los extraescolares, que engloban elementos de carácter psicológico, del entorno social y del familiar; y los escolares, es decir, las prácticas en el aula. De estas últimas, una de las principales es la de la lectura, que genera nuevos conocimientos o deficiencias en el aprendizaje al restringirse a la decodificación de la información por deficiencia en la lectura de comprensión.

\section{Hipótesis particulares}

- Los bajos rendimientos en los estudiantes de la Facultad de Economía están vinculados con problemas de comprensión de los textos. Sin ser la comprensión de lectura deficiente la única causa, es una de importancia relevante.

- Es posible mostrar que la deficiencia en la comprensión de lectura da origen a problemas en la capacidad de aprendizaje mediante la correlación entre los niveles de comprensión lectora y los niveles de avance o rezago académico de los estudiantes de primer semestre.

- A través de la aplicación de encuestas, entrevistas y pruebas de evaluación de la comprensión lectora, puede explorarse la capacidad del estudiante para utilizar la lectura como instrumento de aprendizaje, así como las prácticas o estrategias que le permiten alcanzar el nivel de comprensión.

- Existen varios factores que influyen en la capacidad del estudiante para alcanzar la comprensión lectora. 


\section{Metodología}

Para probar las hipótesis antes señaladas se llevaron a cabo las siguientes acciones:

- Se analizó con detalle la información derivada de los diagnósticos de la DGEE de la UNAM para dos generaciones de la Facultad de Economía.

- Se elaboró un cuestionario con el propósito de detectar tres aspectos principales: nivel de comprensión de lectura, factores relacionados con la comprensión de lectura y trayectoria escolar del estudiante (materias aprobadas o reprobadas, calificación).

- Una vez realizados algunos ajustes en los cuestionarios, se aplicaron, primero, a un grupo piloto de estudiantes y, posteriormente, a una muestra de 228 estudiantes de una generación. La intención inicial fue hacerlo con dos generaciones, pero la información de la DGEE, que fue el punto de partida, sólo permitía realizar comparaciones para la generación 2013.

- Con la información que fue posible obtener de la propia Facultad, respecto a las calificaciones de los alumnos de esa generación, se complementó el análisis de la relación entre los niveles de comprensión de lectura al ingresar a la Facultad y su situación escolar en los dos últimos semestres (enero-mayo y agosto diciembre de 2013, es decir, un año después de su ingreso.

- Se procesó la información de los cuestionarios aplicados para obtener una base de datos que pudiera someterse a la aplicación de los procedimientos y las pruebas necesarios para validar la información.

En la última parte se tuvieron que enfrentar varios problemas que retrasaron la aplicación de los cuestionarios y no permitieron obtener el número previsto de los mismos. La aplicación se concluyó hasta después del 23 de mayo de 2014 y sólo se pudieron obtener 160 de los 228 cuestionarios previstos. De estos, 113 fueron validados para su análisis.

\section{LA LECTURA Y EL PROCESO COGNITIVO}

Flavel y Markman ${ }^{5}$ consideran que el lenguaje escrito y el oral son los principales instrumentos para lograr la adquisición de conocimientos, de forma que la mayor habilidad para la lectura de comprensión propicia también las habilidades para adquirir nuevos conocimientos.

5 Citado por Ramos Maldonado (2005,p. 52). 
Cimaz $(1978)^{6}$ señala que "la adquisición de información es de dos a cuatro veces más rápida por medio de la lectura que de la audición".

Respecto al proceso cognitivo y su vinculación con la lectura, Del Val ${ }^{7}$ señala que "desde el punto de vista psicológico, la lectura y la escritura son procesos complejísimos, (...), y en ellos se originan muchas de las dificultades en el aprendizaje".

Para Delors ${ }^{8}$ la lectura, además de permitir el aprendizaje escolar, es fundamental para aprender a lo largo de la vida. El proceso de comprensión lectora implica la interacción entre el lector y el texto, mediante la cual se incorpora nueva información al esquema cognitivo del sujeto, de acuerdo al propósito con que se lee, dándole una interpretación propia y generando nuevo conocimiento. ${ }^{9}$

Por mucho tiempo se consideró a la habilidad para realizar una buena decodificación como comprensión lectora, pero estudios recientes han demostrado la complejidad del proceso lector y que una fluida decodificación no garantiza la comprensión (Madero Suárez y Gómez López, 2013).

\section{EL PROCESO DE COMPRENSIÓN LECTORA}

\section{Bases psicofisiológicas de la lectura}

Solovieva y Quintanar Rojas (2011, pp. 25-31) señalan que en la organización de la lectura, a nivel psicofisiológico, intervienen diferentes sectores cerebrales actuando como un sistema funcional. Cada sector realiza una actividad específica denominada factor neuropsicológico, que implica desde la identificación de los fonemas hasta el análisis y síntesis de la lectura, pasando por la organización secuencial y la retención de las ideas involucradas en un texto.

Los sistemas funcionales son dinámicos, se modifican con las etapas de desarrollo del ser humano. En la etapa inicial, la actividad principal consiste en un conjunto de acciones voluntarias y conscientes que permita identificar, relacionar y dar significado a los morfemas y fonemas. A medida que avanza el desarrollo cerebral, las actividades de los distintos sectores se automatizan hasta adquirir un nivel de semiconciencia o de conciencia tal que la actividad de varios sectores se reduce.

6 Ibid., p. 56, en nota de píe de página.

7 Ibid., p. 52.

8 Citado por Madero Suárez y Gómez López (2013).

9 Ibid., p. 115. 
Así, las dificultades en el proceso lector pueden tener como causa la falta de madurez de los sistemas funcionales, es decir, fallas por problemas neurológicos relacionados con la génesis morfológica del cerebro. Pero, si un niño enfrenta dificultades en la etapa en que inicia el aprendizaje de la lectura se encuentra morfológicamente sano, estas dificultades pueden estar relacionadas con condiciones desfavorables del medio social que pueden estar generando retraso en el sistema funcional.

De acuerdo con Vigotsky: ${ }^{10}$ "la edad cronológica no se corresponde necesariamente con la edad psicológica y para establecer esta deben considerarse aspectos clave: la situación social, las neo formaciones básicas de la edad y la línea general de desarrollo".

En esta perspectiva, se concede mayor importancia a los factores contextuales en el desarrollo del niño. Así, en el proceso lector, si existe una adecuada guía, es decir, con la intervención de expertos, los niños pueden lograr avances superiores en su desarrollo psicofisiológico. Sin embargo, no se niega que existan condiciones básicas para el logro del objetivo, en este caso la comprensión lectora.

Algunas de las condiciones presentes en las dificultades para la adquisición de la habilidad lectora, señaladas por Solovieva y Quintanar Rojas (2011, pp. 32-38) son: el retardo en la maduración de las estructuras cerebrales; la ausencia de relaciones funcionales; un desarrollo anatómico funcional que no coincide con las exigencias escolares, y un desarrollo insuficiente por falta de estímulo. Las dos últimas tienen como causa los defectos en la enseñanza. Uno de los más comunes es el inicio de la enseñanza de la lectura antes de que se haya completado el desarrollo de los factores neuropsicológicos voluntarios, mismo que inicia en la etapa preescolar, es por eso que esta etapa adquiere particular importancia y requiere una atención especial, pues prepara al niño para los procesos más complejos a que se enfrentara al iniciar la etapa escolar, donde las actividades son menos lúdicas y más estrictas.

Bigge y Hunt (2007) señalan que entre los nueve y los once años el niño debe haber adquirido las habilidades lectoras, en escritura y matemáticas, necesarias para adquirir mayores conocimientos. Puede, entonces, establecer relaciones entre diferentes áreas del conocimiento y utiliza el lenguaje como medio de comunicación, pero también como instrumento de cognición. 


\section{Etapas y niveles en el proceso de la lectura}

Para lograr el paso de la decodificación a la comprensión de la lectura, es preciso cubrir diferentes etapas o niveles que, de acuerdo a la teoría transaccional, dependen de varios factores. ${ }^{11}$ Los tres componentes principales son: el lector, el texto y el contexto. En el caso del lector destaca la importancia de la motivación, los procesos cognitivos y las experiencias de vida que han tenido lugar en el individuo. Ambos desarrollados en un entorno o contexto que posibilita, o no, que un estudiante logre no sólo decodificar, sino pensar y elaborar el contenido para hacerlo propio e integrarlo a su acervo de conocimientos. Por otra parte, las características del texto, como tema, autor, extensión, presentación, etcétera, permiten la conexión entre la motivación y las características del lector que lo hace más o menos accesible y posibilita que el proceso de identificación y cognitivo propicie la decodificación y reconstrucción de significados.

De acuerdo a lo anterior, no existen entonces respuestas únicas ante la lectura de un texto. El continuum, desde el propósito hasta la percepción de estados afectivos en el proceso de la lectura, atraviesa por varias instancias: la postura del lector de acuerdo a sus objetivos, es decir, la motivación para la lectura, o lectura eferente; los saberes, experiencias y creencias movilizados en el transcurso de la lectura, y, finalmente, las interpretaciones, explicaciones y sentimientos o emociones que se provocan en el lector.

Desde otra perspectiva, sólo el proceso de decodificar implica varias etapas que permiten transitar de la identificación de los patrones gráficos a la integración del significado de las frases de un texto, así como hacia la comprensión. Para Alonso Tapia (2005) son seis. ${ }^{12}$ Muñoz-Valenzuela y Scheltraete $(2008$, p. 3) consideran tres niveles: en el primero, el nivel superficial, se distinguen las palabras y la sintaxis del texto; en el segundo, denominado base del texto, se decodifican los significados o semántica del texto, y el tercero, el modelo de situación, implica un procesamiento más elaborado del texto, donde entran en juego los conocimientos, las experiencias y la capacidad del lector para establecer comparaciones y relaciones, y aplicar a situaciones distintas la información que proporciona el texto.

11 De acuerdo con Rosenblat, citado por Rapetti y Vélez (2012).

12 Cuatro fases para lograr el significado de las frases, unapara la integración de las frases de los distintos ciclos de la lectura, dos para obtener el significado global del texto, integrando coherentemente las diferentes ideas que contiene (pp. 65-76), y una que implica un nivel de lectura superior, donde se identifica la intención del autor. 
En general, se consideran entre tres y cinco niveles de lectura, dependiendo de la desagregación o agregación de capacidades. Consideramos aquí la división en tres niveles: el literal, el inferencial y el analógico-crítico. Cada uno, a su vez, comprende un conjunto de acciones o actividades realizados por el lector.

Como puede observarse en el cuadro 1, sólo el proceso de decodificación o de recuperación de la información es complejo, porque depende de condiciones psicológicas, cognitivas y del entorno, que posibilitan o no generan un buen decodificador. Pero ésta, aun siendo un paso necesario, es insuficiente para la comprensión, constituye sólo una de las variables que intervienen en el proceso. La comprensión lectora requiere de una elevada cantidad de elementos cognitivos.

Entre los investigadores existe acuerdo en que la complejidad del proceso de comprensión lectora tiene una connotación individual y, por tanto, da lugar a diferencias importantes en los niveles que se alcanzan. También hay acuerdo en que la enseñanza de la lectura con frecuencia se enfoca a lograr la fluidez y rapidez necesaria para una adecuada decodificación, pero es menos frecuente que se insista en el procesamiento de los textos hasta alcanzar plenamente la comprensión lectora, es decir, alcanzar el tercer nivel.

\section{LA COMPRENSIÓN LECTORA EN MÉXICO}

La mayoría de los autores que han analizado el caso mexicano concluyen que la estructura del sistema educativo en el nivel primario incorpora como preocupación central la lectura en los primeros tres grados, pero no se alcanza el nivel de comprensión lectora.

"El beneficio de la educación es su efecto duradero; si el efecto es escaso podemos asegurar, sin temor a equivocarnos, que ha sido un fracaso", señalanBigge y Hunt (2007, p. 18). Si con esta idea examinamos los resultados obtenidos en el sistema educativo mexicano, no quedaría más que coincidir con los autores. Y el problema no es menos cuando sabemos que la mayor proporción de estudiantes del país, en todos los niveles, es atendida por instituciones públicas.

En los últimos años se ha repetido en diferentes medios y foros que el sistema educativo muestra serias deficiencias, que es imprescindible realizar cambios de distinta naturaleza. Así, en 2013 se aprobó la Reforma Educativa del gobierno federal, reforma que desde sus inicios contó con muchas aristas difíciles que han generado múltiples inconformidades. 


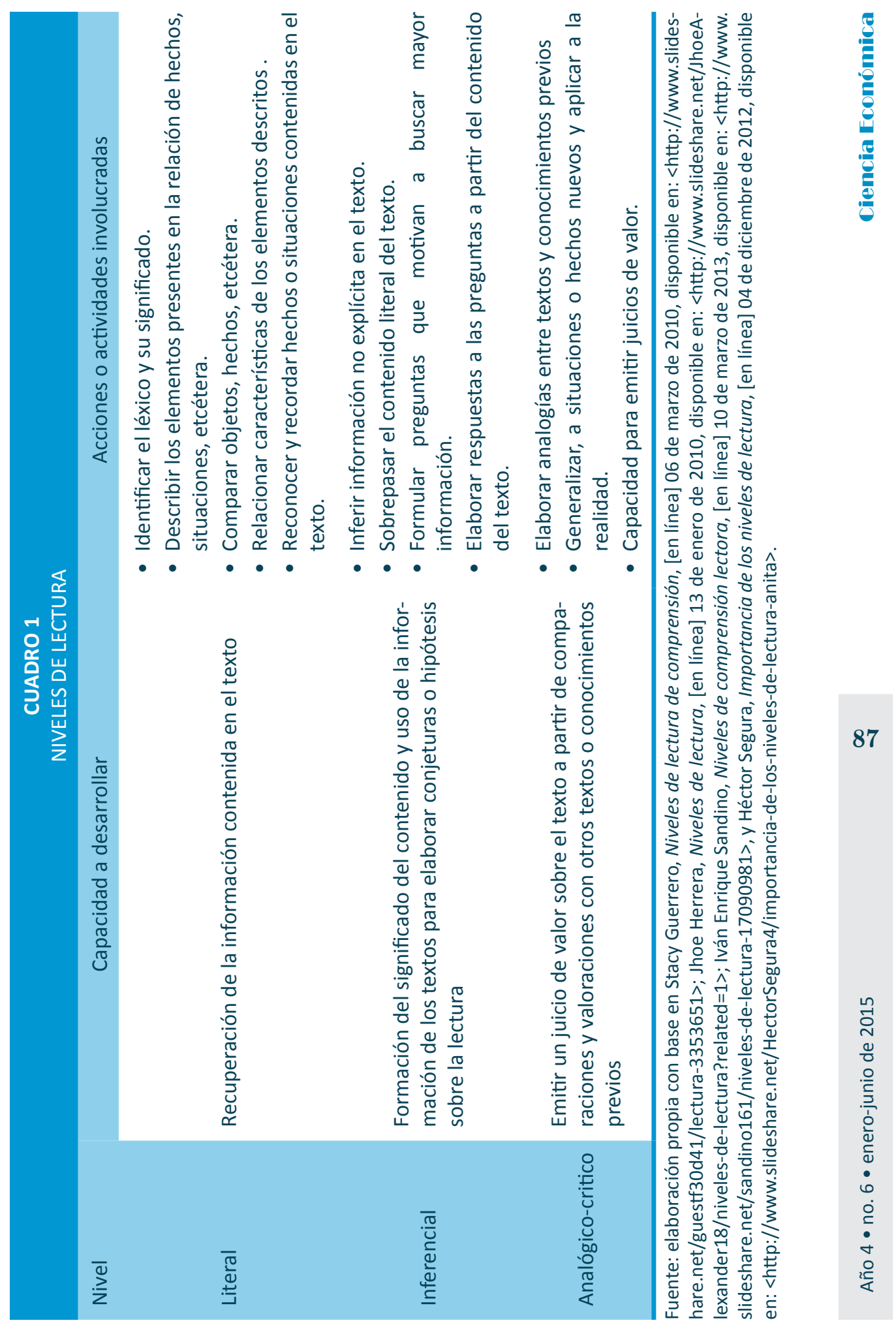


En octubre del mismo año, en un periódico de publicación nacional, apareció una nota respecto a la valoración que hace el World Economic Forum, a través del Índice del Capital Humano, sobre el sistema educativo mexicano: "El índice, que tiene la finalidad de identificar a los países que están en mejores condiciones de transformar las inversiones en salud, educación y desarrollo de aptitudes, en una ventaja económica, ubicó a México en el lugar 102 en materia de calidad del sistema educativo, de una lista de 122 países, en donde el número uno es el mejor y el 122 el peor" (Gazcón, 2013).

Dicha situación se evidencia con los resultados de los exámenes de Evaluación Nacional del Logro Académico en Centros Escolares (ENLACE) para 2012, que para el caso de lamateria de español son los siguientes: en la primaria general pública, $17.7 \%$ de los alumnos en $6^{\circ}$ de primaria mostraron conocimientos insuficientes; $42 \%$, elementales; $32 \%$, buenos, y poco más de $8 \%$ excelentes. Esta situación tiene en otros ámbitos educativos resultados todavía más preocupantes: en el Consejo Nacional de Fomento Educativo (CONAFE) se reporta un porcentaje de insuficiencia de $43.2 \%$, y en la educación para indígenas de $38.1 \%$; en tanto que el porcentaje para conocimientos elementales es de 46.2 y 43.8 por ciento respectivamente.

A nivel secundaria los resultados son peores aún: en el sistema general casi $42.2 \%$ calificó como insuficiente, $41.4 \%$ como elemental, $15.5 \%$ como bueno y sólo un $0.9 \%$ como excelente. Estos resultados también indican una mayor deficiencia en sistemas de secundaria diferentes como la secundaria técnica y la telesecundaria, donde los porcentajes de insuficiencia se elevan a 46.1 y 46.8 por ciento respectivamente. ${ }^{13}$

En otras áreas de conocimiento, como matemáticas y ciencias, las cifras muestran también claras deficiencias en los sistemas educativos públicos. Pero nos centraremos sólo en español, y en particular, en la lectura de comprensión, puesto que el lenguaje es la primera y más importante herramienta que tiene el hombre para acercarse al conocimiento, para aprender y generar nuevo conocimiento. Primero, el materno y, posteriormente, los lenguajes de mayor uso para el desarrollo científico, como el algebra o algún idioma extranjero.

El problema de las deficiencias es que, desafortunadamente, se arrastran hacia los niveles de estudio superior, e incluso en el posgrado. En la UNAM desde hace algunos años se aplican exámenes de diagnóstico en matemáticas, español, inglés y conocimientos generales a los estudian-

13 ENLACE, Estadísticas nacionales 2006-2012. [en línea] Disponible en: <http://www.enlace. sep.gob.mx/content/ba/pages/estadisticas/estadisticas_2013.html>. 
tes de primer ingreso. Los resultados muestran que existe un problema importante en las dos primeras. Del total de alumnos de nuevo ingreso, una proporción importante tiene niveles muy bajos en el manejo de español y de matemáticas.

Para algunas carreras puede considerarse que tener un buen nivel de cualquiera de estas asignaturas no es imprescindible, pero cuando existen problemas en el uso del lenguaje es posible que se convierta en uno de los principales factores relacionados con las habilidades requeridas para la comprensión de los textos, limitando seriamente los niveles de aprendizaje en diferentes áreas del conocimiento.

Así, la incapacidad para leer y, más aún, para entender los libros de texto en cualquier asignatura podría estar generando incapacidad para aprender ciencias, matemáticas, geografía o historia.

Las causas de esta situación son diversas. Para algunos, el contenido de los programas de estudio en la materia de español es demasiado extenso y compite, además, con los contenidos del resto de las materias. Otros señalan también las limitaciones de los profesores en este campo.

Independientemente de las causas, lo cierto es que la percepción de deficiencias en el proceso de lecto-escritura persiste en el ámbito universitario. La expresión general de muchos de los docentes es "los alumnos no leen y cuando lo hacen no entienden".

Esta frase, no literal, pero que resume lo que es una opinión común entre los docentes, puede confrontarse con los resultados de diferentes evaluaciones, como la que lleva a cabo la UNAM y cuyos resultados, como ya se ha señalado, muestran deficiencias en español y matemáticas para una parte importante de los estudiantes de primer ingreso.

A partir de lo anterior y con la intención de profundizar en la relación comprensión lectora-aprendizaje, el presente documento busca encontrar algunos elementos que permitan concretar y ampliar la información general al respecto en un caso particular, el de la Facultad de Economía de la UNAM. El caso fue elegido por dos razones fundamentales: la cercanía y la experiencia en el ámbito docente en ese lugar y, por tanto, la posibilidad de acceder a información relevante; la otra es que en esa Facultad se atraviesa por un proceso de revisión curricular y los resultados de esta investigación pueden ser útiles para el proceso.

\section{LA COMPRENSIÓN LECTORA EN LOS ESTUDIANTES DE LA FACULTAD DE ECONOMÍA}

La Licenciatura en Economía comprende diez semestres, divididos en dos partes: un núcleo básico centrado tanto en la formación de carácter 
teórico-conceptual, donde el objetivo es dotar al estudiante del lenguaje, los métodos y los instrumentos indispensables y comúnmente aceptados en la formación del economista, como en la orientación hacia la aplicación de los fundamentos indispensables de la economía en los diversos ámbitos de la profesión; y un núcleo terminal, conformado por un conjunto de asignaturas cuyo propósito es profundizar en la formación teórica e iniciar la práctica profesional con énfasis en un área específica de la economía.

Del total de asignaturas del Plan de Estudios, cerca de $72 \%$ corresponden al núcleo básico, de las cuales poco más de $37 \%$ involucra el uso de las matemáticas, además del contenido lógico-conceptual de la ciencia económica, este grupo lo denominaremos asignaturas lógico-conceptuales. El resto implica un volumen importante de lecturas, a este grupo nos referiremos como asignaturas histórico-conceptuales.

En el núcleo terminal, conformado por 17 asignaturas, la composición entre asignaturas, donde la mayor dificultad es el volumen de textos y aquellas donde se agrega el uso de las matemáticas, dependerá del área de estudios elegida por el estudiante para su formación terminal. De las seis áreas de especialización, la mitad comprenden niveles de matemáticas complejos. La otra mitad, lecturas con mayor grado de dificultad y capacidad de realizar un análisis de los textos hasta el tercer nivel de comprensión lectora.

Los índices generales de reprobación en núcleo básico indican que entre 44 y 48 por ciento de los estudiantes no acreditan todas las materias pertenecientes al grupo lógico-conceptual. En tanto para las del grupo histórico-conceptual el índice es de 40 por ciento. ${ }^{14}$

Como puede verse, el problema es serio y requiere de un análisis a fondo y en detalle que posibilite implementar las medidas necesarias para afrontarlo.

\section{Características generales de la evaluación en comprensión de lectura de la población objetivo}

Del análisis realizado a la información proporcionada por la DGEE para los estudiantes de primer ingreso de la Facultad de Economía de la UNAM, generación 2013 (ingresados en agosto de 2012), pueden derivarse los siguientes resultados de acuerdo a los distintos niveles de comprensión lectora (véase el cuadro 2).

14 Datos obtenidos del Documento Ejecutivo de Diagnóstico, Facultad de Economía, UNAM, 2013 (mimeo). Documento que forma parte del proyecto de transformación del Plan de Estudios de la Licenciatura escolarizada de la Facultad. 
- Del total de alumnos de la generación 2013 (560), 49.6\% clasifican con un nivel de comprensión de lectura alto y muy alto.

- Poco más de $27 \%$ se ubican en el nivel medio.

- Cerca de $23 \%$ se encuentra en los niveles bajo y muy bajo.

- La mayor proporción de estudiantes de la generación es masculina (68.7\%).

- La distribución por sexos en cada nivel muestra que, en general, predomina también la población masculina, pero con algunas precisiones: el mayor porcentaje de estudiantes femeninas se ubica en el nivel medio y muy bajo, en tanto los hombres tienen mayor presencia en los niveles alto y muy alto.

\begin{tabular}{lcc}
\multicolumn{3}{c}{ CUADRO 2 } \\
& \multicolumn{2}{c}{ COMPRENSIÓN LECTORA POR NIVELES } \\
Clasificación & \multicolumn{2}{c}{} \\
& Número de alumnos & Porcentaje \\
Muy alto & 101 & 18 \\
Alto & 177 & 31.6 \\
Medio & 155 & 27.68 \\
Bajo & 91 & 16.25 \\
Muy bajo & 36 & 6.43 \\
Total & 560 & 99.96 \\
\hline
\end{tabular}

Fuente: elaboración propia con base en información del documento Resultados del Examen Diagnóstico de los alumnos de primer ingreso de la Facultad de Economía, 2012-2013 de la Dirección General de Evaluación Educativa (DGEE), UNAM.

Para la muestra, los resultados del análisis de la fuente antes mencionada pueden observarse en la gráfica 1.

Como puede verse, la distribución del número de alumnos por clasificación guarda características muy similares a la población total, lo mismo sucede respecto a la distribución por género. Se mantiene el predominio de la población masculina en el total de la muestra (67.84\%) y en cada uno de los niveles de comprensión lectora.

De la misma forma, el mayor porcentaje de población masculina se encuentra en los niveles muy alto y alto, mientras la femenina tiene un valor mayor en los niveles medio y muy bajo.

En cuanto a la distribución de los estudiantes de la muestra por turno, podemos ver en la gráfica 2 que la mayor proporción se ubica en el turno matutino. Esta situación se mantiene en cada uno de los niveles de la clasificación de la comprensión lectora, con una excepción importante: del total de hombres del nivel alto, poco más de $67 \%$ se localizan en el 
turno vespertino. También llama la atención que la proporción de varones en el turno vespertino es mayor, en general, que el de mujeres.
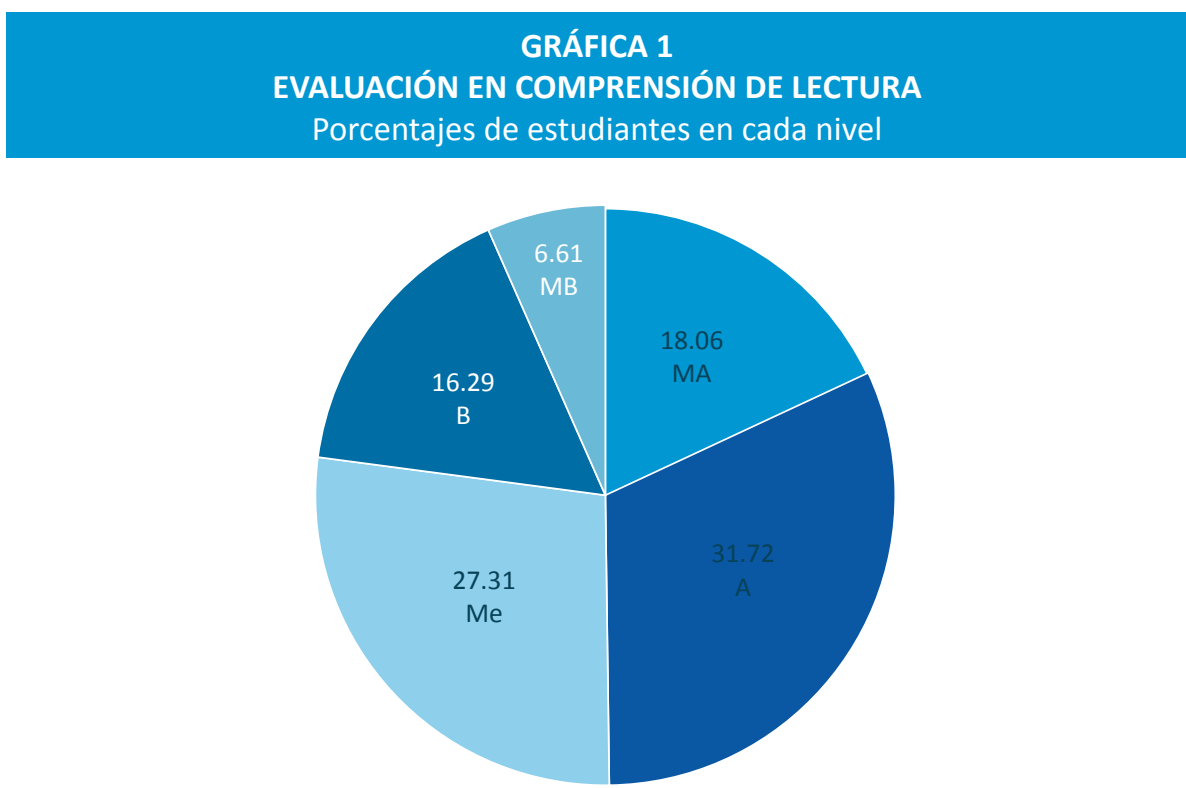

Fuente: elaboración propia con base en la información del documento Resultados del Examen Diagnóstico de los alumnos de primer ingreso de la Facultad de Economía, 2012-2013 de la DGEE, UNAM.
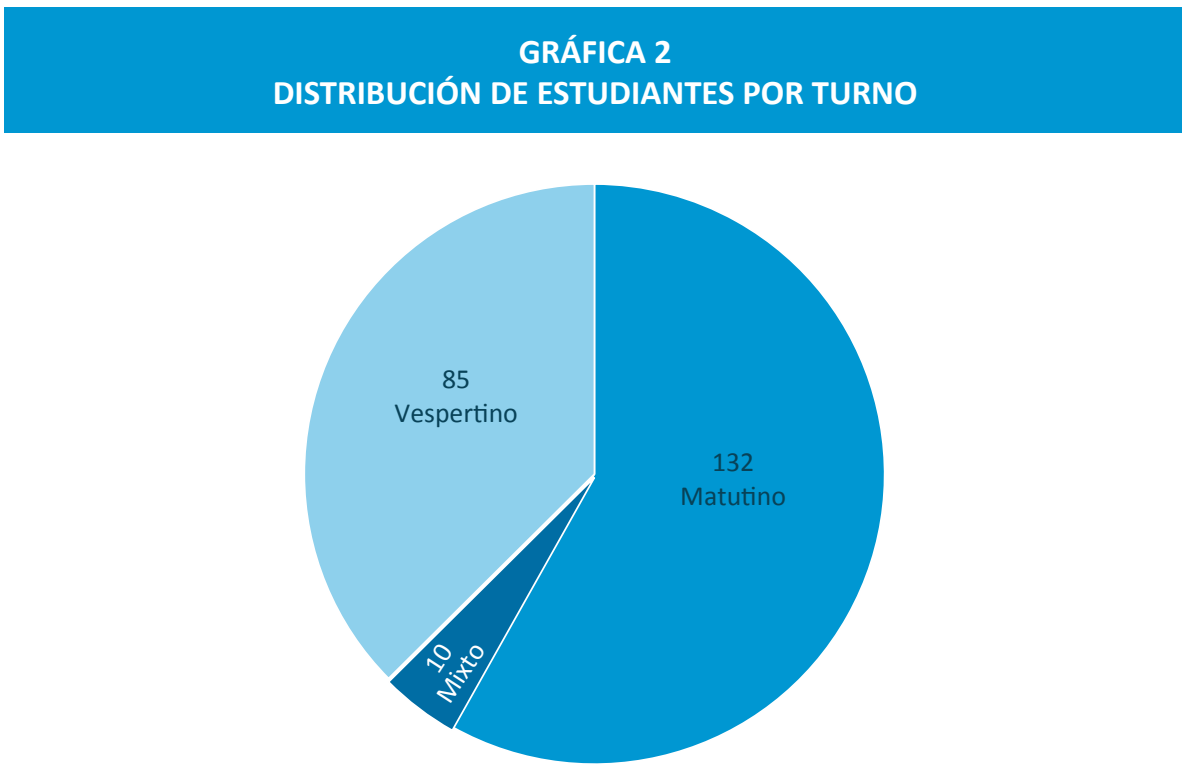

Fuente: elaboración propia con base en la información del documento Resultados del Examen Diagnóstico de los alumnos de primer ingreso de la Facultad de Economía, 2012-2013 de la DGEE, UNAM. 


\section{Relación comprensión lectora-aprovechamiento}

A partir de los datos de la muestra obtenidos de la DGEE y con la información que se logró recopilar en la Facultad de Economía, se integró un cuadro que relaciona las calificaciones de los alumnos de dicha muestra para dos semestres, que corresponden al primero y segundo semestres de los estudios de la Licenciatura en Economía.

Para explicar los resultados, describiremos algunos de los conceptos incluidos en el cuadro.

Nivel o clasificación en la comprensión lectora: se define en función del porcentaje de respuestas en el examen de diagnóstico de la DGEE. Se establecen cinco niveles:

- Muy alto, comprende un rango de 100 a 70 por ciento de respuestas correctas.

- Alto, de menos de 70 a 62.5 por ciento.

- Medio, de menos de 62.5 a 50 por ciento.

- Bajo, de menos de 50 a 37.5 por ciento.

- Muy bajo, de menos de 37.5 a 0 por ciento.

Calificación promedio: es la calificación promedio obtenida en el semestre de asignaturas cursadas y presentadas.

No presentada (NP): se anota de esta manera las asignaturas inscritas pero que, por alguna razón, no fueron concluidas, por lo tanto no se obtiene calificación.

Porcentaje de estudiantes con NP: se anota de esta forma la proporción de estudiantes que en cada nivel tienen una o varias asignaturas no presentadas.

Como puede observarse en el cuadro 3, en términos de calificaciones los niveles alto y medio son los que se encuentran en mejor situación, en tanto los niveles muy alto, bajo y muy bajo aparecen con valores menores que los anteriores, pero similares entre ellos. Esto significaría que las calificaciones no dependen necesariamente del nivel de comprensión lectora. Sin embargo, si analizamos la columna de NP promedio podemos observar que salvo el nivel medio, a menor nivel de comprensión lectora, mayor es el número de NP.

Como ya se explicó antes, la NP se refiere al número de asignaturas que los estudiantes abandonan, pero también se emplea, generalmente, como una estrategia del estudiante para evitar que aparezcan con 
calificación reprobatoria sus asignaturas y cada alumno tiene derecho a cursar dos veces una asignatura. Este elemento puede considerarse como indicador de rezago del estudiante respecto al avance curricular, es decir, en el cumplimiento del Plan de Estudios en los tiempos y forma establecidos.

Si se analiza con más detalle caso por caso, encontramos una tendencia general a que las proporciones de estudiantes con calificaciones mayores a 8 se encuentren en los niveles muy alto y alto. En el nivel muy alto se ubica entre 40 y poco más de $53 \%$ del total de estudiantes en la muestra, y tienen calificaciones mayores a 8. En el nivel alto este grupo se encuentra en una proporción mayor a $30 \%$, pero menor a $35 \%$. Y en el nivel medio el porcentaje de estudiantes con calificaciones mayores a 8 oscila entre 27 y 31 por ciento.

Así, parece comprobarse el hecho de que el nivel de comprensión lectora está relacionado con el grado de aprovechamiento escolar, en términos del avance académico, aun cuando respecto a las calificaciones es poco claro.

\section{Análisis del cuestionario}

Con el fin de detectar los factores que propician o inhiben la comprensión lectora y la relación con el nivel de aprendizaje, se aplicó un cuestionario con preguntas en su mayoría cerradas.

Las primeras preguntas intentan disminuir la tensión al responder el cuestionario. En un segundo apartado, se busca identificar el nivel de dificultad que representa para el estudiante las asignaturas de su Plan de Estudios y las razones para ello. Finalmente, un tercer apartado pretende identificar los problemas en los proceso de la comprensión lectora.

A partir del análisis del cuestionario pueden desprenderse varios resul-tados relacionados con las prácticas lectoras e inferir las posibles relaciones entre estas y los hallazgos expuestos en el apartado anterior.

\section{Características generales}

Del número de estudiantes encuestados, 65.5\% pertenecen al sexo masculino, en tanto $34.5 \%$ son mujeres. La proporción de estudiantes provenientes de bachilleratos públicos es absolutamente mayoritaria (86.7\%). Un $88.5 \%$ tiene 21 años de edad, y $11.5 \%$ son mayores a dicha edad. La mayor parte (79.6\%) realizó sus estudios en los tres años reglamentarios; del resto, en su mayoría lo hicieron en cuatro años. 
Reflexiones sobre la docencia

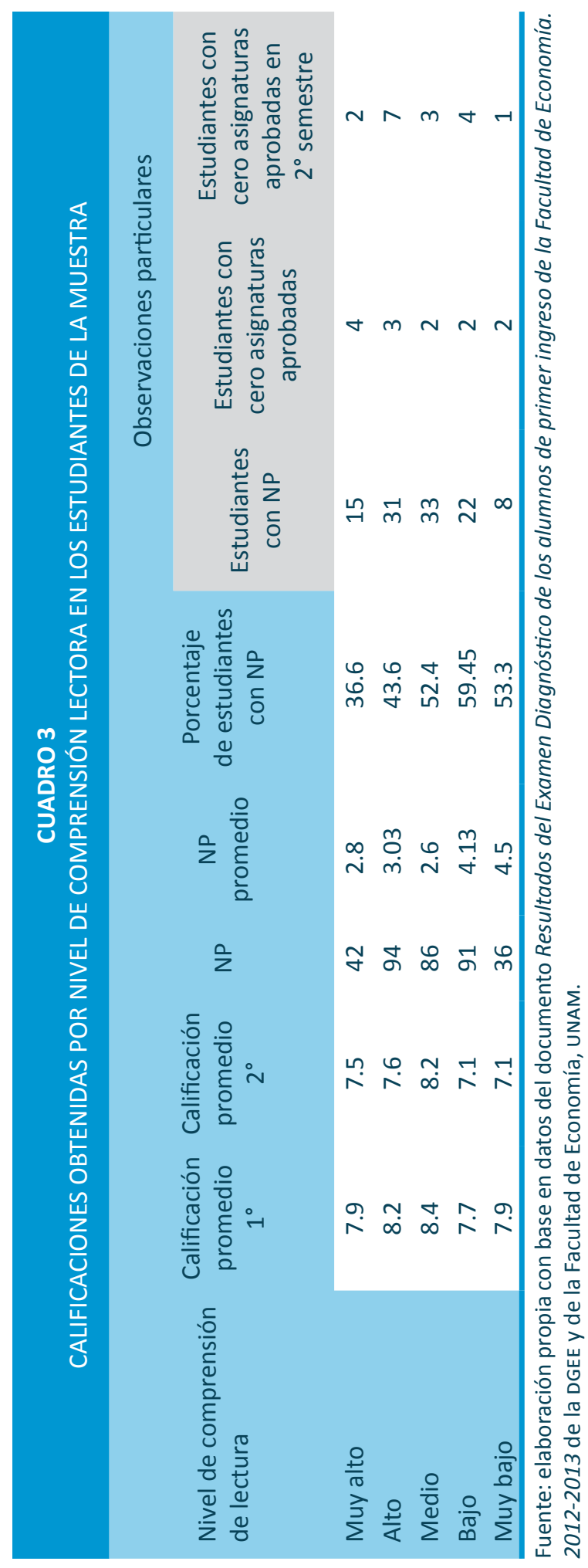

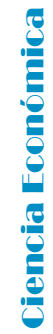




\section{Características relacionadas con la comprensión lectora}

En el cuestionario, las cuatro primeras preguntas proporcionan información general sobre las características de los estudiantes como lectores, en relación con textos no académicos, es decir, respecto a lecturas estéticas.

Nivel de comprensión lectora. De acuerdo con datos obtenidos de la DGEE, del total de estudiantes que contestaron los cuestionarios, $48.7 \%$ obtuvo un nivel de comprensión lectora considerado como muy alto y alto, en tanto que $51.3 \%$ se encuentra ubicado entre los niveles medio y muy bajo; de este grupo, 53\% está en los niveles bajo y muy bajo, lo que significa que $47 \%$ se ubica en el nivel medio.

Destaca en este apartado que la mayor parte de las mujeres (51.3\%) pertenece a los estratos muy alto y alto, en tanto la proporción de hombres es ligeramente menor (48.6\%).

Preferencias lectoras. Esta variable está relacionada con la motivación en la lectura, factor que la mayor parte de los especialistas en el tema de comprensión lectora consideran como fundamental en cuanto a uno de los componentes básicos del proceso lector. ${ }^{15}$ Se trata de medir la amplitud de temas que el estudiante abarca en sus lecturas no escolares, por lo tanto voluntarias, y por ello con un sentido más lúdico pero con impacto en el desarrollo de capacidades.

Indica, en alguna medida, las posibilidades que el lector tiene en cuanto al manejo de vocabulario y variedad de conocimientos, por considerarse un lector consuetudinario de temas diversos. En la respuesta a la pregunta correspondiente, $52.5 \%$ de los estudiantes manifestaron niveles de preferencias por abajo del promedio, es decir, con un manejo reducido de temas y probablemente en vocabulario y temáticas.

Otro elemento importante en la motivación es el criterio o criterios del lector para seleccionar sus lecturas. En este rubro, a mayor número de elementos el valor se incrementa y representa una selección mejor realizada.

Las respuestas a esta pregunta muestran que una mayor proporción (71.7\%) de los estudiantes tienen un valor por debajo del promedio, esto es, utilizan pocos elementos para seleccionar sus lecturas.

15 Como se menciona en el apartado teórico, los componentes básicos del proceso lector comprenden: lector, texto y contexto. 
Número de libros leídos al año. Como parte de los factores que proporcionan al lector las habilidades para mejorar su comprensión lectora tenemos la cantidad de lecturas que se realizan, por esta razón se incorporó una pregunta respecto al número de libros no académicos leídos en el año. En tal sentido, las respuestas indican que en $82.3 \%$ de los casos las lecturas son menores al promedio. Esto está representando un reducido número de lectores habituales.

De acuerdo con Vargas Ascurra (2010), un buen lector debe tener un propósito y realizar una pre-lectura, es decir, un conjunto de acciones antes de llevar a cabo la lectura: hojear el texto para observar sus características; realizar predicciones sobre el contenido, así decide o no realizar la lectura; planifica y hace inferencias conscientes sobre la misma.

Por lo anterior, se cuestionó sobre la existencia de un propósito al iniciar la lectura, $61 \%$ respondió positivamente. De igual forma, se les cuestionó respecto a los criterios de selección en sus lecturas no escolares, con el objetivo de definir si se lleva a cabo una revisión previa del texto a leer; el número de estudiantes con una respuesta inferior al promedio fue de $71.6 \%$, esto expresa que la mayor parte de los estudiantes no llevan a cabo una revisión completa de las características de la lectura antes de hacerla.

Cuando el lector tiene objetivos definidos y las características del texto corresponden con sus expectativas, es posible que lo evocado y provocado con la lectura permita recordar mucho de lo leído. Con la intención de explorar este aspecto se preguntó sobre los aspectos de la lectura recordados (autor, título, tema, personajes, situaciones, etc.); el $59.3 \%$ de los estudiantes obtuvo un porcentaje menor al promedio. Lo anterior significa que la mayoría de los estudiantes tienen una retención deficiente de sus lecturas recreativas, y por lo tanto voluntarias, elemento que debiera resultar favorable para un mejor resultado.

\section{Estrategias y habilidades lectoras en relación a las asignaturas}

En este apartado, el conjunto de trece preguntas se divide de la siguiente forma:

- Una pregunta para el objetivo o propósito de la lectura eferente. ${ }^{16}$

- Una para el método de búsqueda de información.

16 Se considera lectura eferente a la relacionada con un propósito académico o de trabajo, es decir, implica búsqueda de información o de respuesta a preguntas académicas o laborales: no son voluntarias, esto es, generalmente son requeridas por agentes externos al lector. 
- Cinco para las estrategias lectoras (dos de estas en relación con el vocabulario).

- Dos para las estrategias de procesamiento de las lecturas realizadas.

- Dos para tratar de ubicar a los estudiantes en el nivel de comprensión lectora (literal, inferencial o analógico crítico).

Respecto al propósito de lectura, cuando se trata de textos académicos, $76.1 \%$ manifiesta que no siempre es claro. Es decir, sólo un $23.9 \%$ de los estudiantes tienen un objetivo claro al abordar sus textos. En este sentido, es posible que las estrategias para buscar información y realizar las lecturas no sean las correctas.

En cuanto a las estrategias para buscar información en relación con sus asignaturas, las respuestas de los estudiantes se dividen casi en la mitad para valores por arriba del promedio y valores menores al promedio. Se indica de esta forma que existe una misma proporción de estudiantes que siguen varios pasos para lograr la información deseada, que aquella con una visión limitada sobre la forma de alcanzarla.

En un sentido similar la pregunta referente a método de estudio pretende darnos un indicio del uso de distintas formas para estudiar sus asignaturas. Las respuestas muestran un $67.3 \%$ de estudiantes con un reducido espectro de actividades en el estudio, en tanto poco más de $30 \%$ usan estrategias diversas.

Como se señaló arriba, la lectura requiere precisar los propósitos y llevar a cabo actividades de pre-lectura, en las preguntas del cuestionario referidas a ello las respuestas son las siguientes: la proporción de estudiantes con acciones insuficientes para clarificar sus objetivos asciende a $80.5 \%$, en tanto que para las actividades de pre-lectura, también deficientes, la proporción alcanza el 85 por ciento.

Un problema en la comprensión lectora es el manejo de vocabulario. Arriaga Campos (2011), en una investigación para estudiantes de la Facultad de Economía, encuentra que existe pobreza lexicográfica, y señala: “(...) es un hecho incontrovertible que la asimilación de conocimientos específicos requiere de una plataforma léxica y una competencia para decodificación y codificación de textos, sin las cuales los estudiantes recorren los objetivos de los planes curriculares con déficit acumulativos (...)".

En sus resultados se muestra que los estudiantes construyen el $50 \%$ de su discurso en temas económicos con 28 ó 30 vocablos, de los cuales, como elementos nucleares, se encuentran entre 2 y 3 sustantivos y entre 3 y 5 verbos. Estas cifras son similares en la redacción de temas no económicos, lo cual muestra que la formación académica específica no agrega 
vocablos significativos al léxico básico del estudiante. Lo anterior parece indicar deficiencias en la comprensión lectora de sus textos económicos, tal vez producto de los defectos en el proceso lector anterior.

Cuando el lector tiene el hábito de la lectura es factible la comprensión, derivando el significado de los vocablos o conceptos desconocidos a partir del contexto de la lectura, cuando ello no es posible se tiende a acudir a diccionarios. Con el propósito de obtener información respecto al grado de amplitud del vocabulario y de la estrategia, se realizaron dos preguntas. La primera cuestionaba sobre la frecuencia en vocabulario desconocido en sus lecturas académicas y la segunda abordaba la estrategia para resolver lo anterior. La proporción de estudiantes que manifiesta tener problemas con el léxico comprende cerca de $86 \%$; de estos, $84 \%$ tiene un número reducido y poco eficiente de estrategias para afrontar el problema de vocabulario.

Respecto a las estrategias lectoras, la pre-lectura, como ya se mencionó, tiene importancia central para decidir abordar o no un texto, pero también para saber cómo hacerlo. Aquí, $61.2 \%$ de los estudiantes muestra una revisión previa poco cuidadosa de los textos.

Otro paso en la comprensión lectora requiere procesar los textos con fines de obtener la información pertinente y lograr una representación mental del contenido, lo cual implica el nivel de lectura inferencial donde se requiere: aplicar conocimientos previos, establecer analogías, relacionar ideas, complementar información, obtener el significado de vocablos no conocidos mediante el contexto y resumir el contenido. ${ }^{17}$ Para analizar este aspecto, en el cuestionario se pregunta sobre las acciones que el estudiante realiza cuando aborda una lectura respecto a un tema. En este caso, la pregunta comprende cinco acciones básicas, de las cuales sólo $33.6 \%$ de los estudiantes llevan a cabo la mayor parte, el resto se encuentra por abajo del promedio de valor para esta pregunta.

De manera complementaria, se cuestiona respecto a la estrategia para efectuar la representación del texto y recordar su contenido. Las respuestas indican cerca de $70 \%$ de estudiantes con estrategias deficientes.

Para indagar qué proporción de estudiantes logra alcanzar el tercer nivel en la comprensión de la lectura, el analógico-crítico, se formularon dos preguntas. Una en relación con la capacidad crítica, donde se obtuvo una proporción de $18.6 \%$ de estudiantes que manifiestan haber alcanzado este nivel. La otra pregunta se refiere a la habilidad para elaborar un documento útil y claro para su uso como base de estudio, aquí la proporción favorable es de poco más de 28 por ciento. 
Finalmente, para explorar el grado de dificultad que representan las asignaturas y su relación con la lectura, se elaboraron cuatro preguntas; dos referentes al grado de dificultad que representan cinco de las principales materias en la Licenciatura en Economía, las otras dos pretendían encontrar las causas de la dificultad. En cuanto a las asignaturas de mayor facilidad, las respuestas señalan a la Historia en primer lugar, seguida por las Matemáticas y la Teoría Económica. De las consideradas difíciles, en primer lugar se ubican las Matemáticas, después la Teoría Económica y en el tercer lugar la Economía Política.

De las razones esgrimidas para considerar fáciles las asignaturas se mencionaron el gusto por la temática y que resultaban sencillas o más comprensibles para el estudiante. En cuanto a las asignaturas consideradas con mayor grado de dificultad, el mayor número de alumnos anota como razones las siguientes: son confusas o poco comprensibles, difíciles de recordar o aburridas. Pero varios señalan a los profesores como responsables, por la falta de claridad al exponer los temas.

\section{CONCLUSIONES}

La mayor proporción de estudiantes de la generación 2013 de la Facultad de Economía en las evaluaciones realizadas a su ingreso se ubica en los niveles altos y muy altos de comprensión lectora. De acuerdo con estos

100 datos, podrían suponerse altos niveles de aprovechamiento en una proporción semejante.

No obstante, en el análisis de los datos respecto a la relación entre los niveles de comprensión lectora, las calificaciones y el avance curricular nos muestran, en términos de calificaciones, que la relación no parece muy clara; esto es, no son significativamente diferentes las calificaciones de los estudiantes clasificados en los niveles de comprensión lectora más elevados en relación con los comprendidos en los de menor nivel. Pero considerando el avance curricular, los alumnos con mayor rezago en materias acreditadas se corresponden con los niveles bajo y muy bajo de comprensión lectora; en tanto los de menor rezago se ubican en los niveles altos de comprensión lectora.

Lo anterior parece indicar que efectivamente existe relación entre los niveles de aprovechamiento y los niveles de comprensión lectora.

Respecto a los factores determinantes para lograr un tercer nivel de comprensión lectora, en el que se completa el proceso, los datos obtenidos del cuestionario muestran deficiencias tanto en cantidad de lecturas no académicas realizadas anualmente como en los criterios de 
selección de las lecturas y en la revisión previa a la realización de una lectura. Elementos relacionados con las características de un buen lector, ya que le posibilitan una mayor retención del contenido de las lecturas y lo capacita, además, para el acceso a nuevos conocimientos.

En cuanto a sus textos académicos, donde la lectura es eferente y existe un propósito predeterminado por las necesidades de formación en sus distintas áreas, las deficiencias se enfatizan. Los objetivos no son claros, las estrategias son reducidas y las capacidades adquiridas con las lecturas no permiten afirmar que se ha alcanzado el nivel superior en la comprensión lectora.

A reserva de encontrar nuevas evidencias, podemos decir, en general, que las carencias en la comprensión lectora son un factor explicativo importante de los bajos índices de aprobación en aquellas asignaturas que requieren de la capacidad analógico-crítica, incluido el manejo de lenguajes diversos.

\section{BIBLIOGRAFÍA}

Alonso Tapia, J., 2005. Claves para la enseñanza de la comprensión lectora. Revista de Educación, número extraordinario, pp. 63-93.

Arriaga Campos, R., 2011. Radiografía léxico conceptual del estudiante de Economía. Resultados de la "Radiografía" léxico conceptual de economistas en formación. Introducción. Léxico y Economía. [blog] Disponible en: <http://radiografialexicoconceptualeconomia.blogspot. mx/2011/11/resultados-de-la-radiografia_08.html>.

Bigge, M.L. y Hunt, M.P. 2007. Bases psicológicas de la educación. Reimpresión. México: Editorial Trillas.

Gazcón, F., 2013 (02 de octubre). Educación mexicana, en el sótano; revelan estudios del World Economic Forum. Excélsior (Especiales). [en línea] Disponible en: <http://www.excelsior.com.mx/nacio$\mathrm{nal} / 2013 / 10 / 02 / 921394>$.

Hernández Sampieri, R., Fernández Collado, C. y Baptista Lucio, P., 2010. Metodología de la investigación. 5a edición. México: McGraw-Hill.

Madero Suárez, I.P. y Gómez López, L.F., 2013. El proceso de comprensión lectora en alumnos de tercero de secundaria. Revista Mexicana de Investigación Educativa, 18(56), pp. 113-139.

Muñoz-Valenzuela, C. y Schelstraete, M.-A., 2008. Decodificación y comprensión de lectura en la edad adulta: ¿una relación que persiste? Revista Iberoamericana de Educación, 45/5, 25 de marzo, 18 pp. 
Ramos Maldonado, F., 2005. Pedagogía de la lectura en el aula. Guía para maestros. 4a reimpresión. México: Editorial Trillas.

Rapetti, M. y Vélez G., 2012. Leer para aprender y aprender a leer en la universidad: entre las lecturas estéticas y eferentes. Revista Iberoamericana de Educación Superior, III(7), mayo-agosto, pp. 113-128.

Salgado García, E., 2012. Enseñanza para la comprensión en la educación: la experiencia de una universidad costarricense. Revista Iberoamericana de Educación Superior, 3(7), pp. 34-50.

Solovieva, Y. y Quintanar Rojas, L. 2011. Enseñanza de la Lectura. Método práctico para la formación lectora. México: Editorial Trillas, México.

Tarazón Ruíz, F.A. y Gutiérrez Rohán, D.C., 2013. ¿Qué leen los profesores de la Universidad de Sonora? Un estudio sobre la lectura como práctica cultural en profesores. Revista Iberoamericana de Educación Superior, 4(9), pp. 63-75.

Vargas Ascurra, H.W., 2010. Elementos que articulan el proceso de comprensión lectora. [en línea] 18 de diciembre. Disponible en: <http:// es.slideshare.net/infoudch/elementos-que-articulan-en-el-procesode-comprensin-lectoras. 